\section{P06 THE EPIDEMIOLOGY OF TYPE 1 DIABETES IN CHILDREN FROM NORTHEAST ENGLAND}

doi:10.1136/jech.2010.120477.6

${ }^{1} \mathrm{R}$ J $\mathrm{O}$ McNally, ${ }^{1} \mathrm{~S}$ Court, ${ }^{1} \mathrm{P}$ W James, ${ }^{1} \mathrm{R}$ Pollock, ${ }^{1} \mathrm{~K}$ Blakey, ${ }^{2} \mathrm{M}$ Begon,

${ }^{3} \mathrm{~T}$ D Cheetham. ${ }^{1}$ Institute of Health and Society, Newcastle University, Newcastle upon Tyne, UK; ${ }^{2}$ School of Biological Sciences, Liverpool University, UK; ${ }^{3}$ nstitute of Human Genetics, Newcastle University, Newcastle upon Tyne, UK

Objective Environmental factors are involved in the aetiology of type 1 diabetes. A particular role for infectious exposures has been postulated. Temporal and spatial variation in incidence would be consistent with this hypothesis. We aimed to test predictions of increasing incidence and spatial variation occurring among cases of type 1 diabetes in children (aged $0-14$ years) that might arise as a result of environmental mechanisms.

Design Population-based descriptive analysis of type 1 diabetes data. Setting Northeast England.

Participants The study analysed 545 cases of type 1 diabetes diagnosed in children who were resident in a geographically defined region of northeast England during the period 1990-2007.

Main Outcome Measures Age-specific and age-standardised incidence rates were calculated. Temporal trends were analysed using Poisson regression. Relationships between incidence rates and small area (census ward) population density and Townsend deprivation index (and its components) were analysed using negative binomial regression.

Results Age-standardised incidence rates increased from 15.7 per 100000 population in $1990-1995$ to 27.9 per 100000 population in 2002-2007. Furthermore, there was a regular 6-year cyclical pattern of plus or minus $25 \%$ in incidence rates (RR 1.25 ; $95 \%$ CI 1.11 to 1.41) and an overall increase of $4.8 \%$ per annum ( $95 \%$ CI 3.1 to 6.6). Lower incidence was associated with residence in wards that had higher levels of unemployment ( $R R$ per one percent increase in unemployment $0.97 ; 96 \%$ CI 0.95 to 0.99 ).

Conclusions The results are consistent with the involvement of one or more environmental exposures in aetiology. A possible role for a specific infectious agent should be considered.

\section{P07 IMPROVING THE QUALITY OF DIABETES CARE: EXPLORING THE PERSPECTIVES OF GENERAL PRACTITIONERS IN IRELAND}

doi:10.1136/jech.2010.120477.7

${ }^{1} \mathrm{~S}$ McHugh, ${ }^{2} \mathrm{M}$ O'Mullane, ${ }^{1} \mathrm{I} \mathrm{J}$ Perry, ${ }^{2} \mathrm{C}$ P Bradley, on behalf of the National Diabetes Register Project (NDRP). ${ }^{1}$ HRB PhD Scholars Programme in Health Services Research, Department of Epidemiology \& Public Health, University College Cork, Cork, Ireland; ${ }^{2}$ Department of General Practice, University College Cork, Cork, Ireland

Objective Health professionals are key players in the success or failure of quality management depending on their willingness to accept and adapt to changes in practice. The aim of this study was to explore GPs' experiences of providing diabetes care and their thoughts on the factors affecting its development.

Design Semi-structured qualitative interviews were conducted with GPs to explore their experience of care provision in Ireland, opinions on the factors influencing the development of care and their attitude to registration and audit. The topic guide was informed by the findings of a preceding national survey on the organisation of diabetes care. Analysis was conducted using a pragmatic approach drawing on the Framework method.

Participants A purposive sample of 30 GPs was selected based on 3 sets of inclusion criteria; (a) location (rural/urban), (b) computerised/non-computerised practice, (c) single-handed/group practice.
Results Preliminary analysis has identified both convergent and divergent themes. There were varying attitudes as to how diabetes care should be integrated between primary and secondary care settings. The locus of care varied across participant experiences from primary care-based management to supplementing hospital-led care. Lack of access to auxiliary services and resources were among the common barriers to an effective community-based diabetes service. Those GPs who have developed a structured approach to diabetes care tended to do so as a "labour of love"; most doing so without direct recompense. There were mixed attitudes towards the development of a national register with some questioning its use and benefit at a practice level. A number of GPs felt a national register should to be tailored to meet the needs of health professionals and patients, and not solely used as a research tool. There was ambivalence towards efforts to improve care based on previous experience in the Irish health setting. When asked about the future of diabetes care, GPs proposed regional diabetes management clinics and hoped for greater recognition of the contribution of general practice to diabetes care

Conclusion Preliminary findings suggest there is a typology of care models in existence in Ireland with varying levels of involvement by GPs and diverse access to services. There were tentative attitudes towards quality improvement initiatives including the establishment of a register suggesting a sense of inertia towards organisational change. Extending high quality care to all patients with diabetes can no longer rely solely on the interest of care providers and may require commitment systems-level change.

\section{Cancer \\ P08 CANCER OF OESOPHAGUS OR GASTRICUS-NEW ASSESSMENT OF TECHNOLOGY OF ENDOSONOGRAPHY: FINDINGS}

doi:10.1136/jech.2010.120477.8

${ }^{1,2}$ I Russell, ${ }^{3} \mathrm{~S}$ Attwood, ${ }^{4} \mathrm{H}$ Barr, ${ }^{1} \mathrm{R}$ Tudor Edwards, ${ }^{1} \mathrm{~A}$ Gliddon, ${ }^{1} \mathrm{D}$ Ingledew, ${ }^{5} \mathrm{~K}$ Park, ${ }^{1} \mathrm{D}$ Russell, ${ }^{1} \mathrm{~S} T$ Yeo, ${ }^{1} \mathrm{R}$ Whitaker, on behalf of the COGNATE trial team. ${ }^{1}$ Bangor University, Bangor, UK; ${ }^{2}$ Swansea University, Swansea, UK; ${ }^{3}$ Northumbria Healthcare Trust, North Tyneside, UK; ${ }^{4}$ Gloucestershire Hospitals Trust, Gloucester, UK; ${ }^{5}$ University of Aberdeen, Aberdeen, UK

Objectives Endoscopic ultrasound (EUS) is recommended for staging gastro-oesophageal cancers but has never been rigorously evaluated. This trial assessed whether EUS, when added to usual staging tests: changes treatment; improves survival and quality of life; and uses resources cost-effectively.

Methods We conducted a pragmatic randomised trial in eight centres. As $80 \%$ of participants came from two centres, we combined the other centres for analysis. Patients diagnosed with gastro-oesophageal cancer received a standard staging protocol, after which the multi-disciplinary team agreed a provisional management plan. In principle the choice lay between endoscopic mucosal resection, immediate surgery, neoadjuvant chemotherapy followed by surgery, or multi-modal treatment (ie chemotherapy and radiotherapy). We then randomised consenting patients without metastases by telephone to receive EUS or not. Thereafter we recorded changes in the management plan, in the use of healthcare resources and in participant-reported outcomes. In particular we focused on three facets of quality of life: generic (measured by the EQ5D), cancer-related (FACT general scale and subscales) and conditionspecific (FACT additional concerns scale). We then followed participants at defined intervals till the end of the trial-that is for between one and 3.5 years.

Findings We randomised 223 patients, of whom 213 yielded enough data for primary analysis. At the end of the trial $45 \%$ of EUS 